\title{
Coronary CT angiography offers further risk stratification in the management of patients with normal SPECT results
}

\author{
Matthew J. Budoff, MD, FACC, and Yalcin Hacioglu, MD, EC
}

\section{See related article, pp. 45-51}

Risk stratification strategies used in the diagnosis and management of coronary artery disease (CAD) aim to identify patients with intermediate to high likelihood of significant CAD that might benefit from coronary revascularizations and/or aggressive medical therapy. Myocardial perfusion imaging (MPI) has been an integral part of the traditional risk stratification algorithm for more than two decades. While the presence of significant ischemia on MPI usually necessitates further evaluation with coronary catheterization, patients with normal or low risk scans are mostly managed medically. Even though a normal MPI result generally indicates a benign prognosis, the "warranty period" of such result could be substantially shorter in patients with certain clinical risk factors such as adenosine stress (due to inability to exercise), increasing age, diabetes, female gender in diabetics, and history of known CAD indicating a lower negative predictive value for MPI in this subset of high risk patients. ${ }^{1,2}$

Imaging modalities such as coronary artery calcium scanning (CACS) and coronary CT angiography (CTA) provide information about the coronary anatomy and histology rather than the coronary function. Coronary artery calcium is a marker of vascular injury and its amount (CACS) correlates closely with atherosclerotic burden. ${ }^{3,4}$ Along its comparable diagnostic accuracy in prediction of significant $\mathrm{CAD}$, CACS has also been shown to have significant incremental prognostic value

From the Division of Cardiology, Department of Medicine, HarborUCLA Medical Center, Torrance, CA.

Reprint requests: Matthew J. Budoff, Division of Cardiology, Department of Medicine, LA BioMed Research Institute at HarborUCLA Medical Center, 1124 Carson St, E5, Torrance, CA 90502; mbudoff@labiomed.org.

J Nucl Cardiol 2010;17:13-5.

$1071-3581 / \$ 34.00$

Copyright (C 2009 The Author(s). This article is published with open access at Springerlink.com

doi:10.1007/s12350-009-9176-8 over MPI in several recent studies suggesting a strong potential for its complementary use with MPI. ${ }^{5,6}$ On the other hand, CTA has proven to provide highly accurate diagnosis of obstructive coronary plaques significantly outperforming MPI in all of the studies. ${ }^{7-9}$ CTA also presents more definitive information about the stage of atherosclerotic process by further characterizing the plaques as non-calcified (soft), mixed or calcified, which may also add more value in the prognostication of CAD. There is already some early evidence to support this independent incremental prognostic value for CTA over MPI. ${ }^{10,11}$ It also seems that traditional clinical risk assessment tools of Framingham Risk Score and Adult Treatment Panel III (ATP III) alone cannot accurately predict the atherosclerotic plaque burden as determined by CTA. ${ }^{12}$ This indicates CTA can identify a distinct subset of patients who would have been misdiagnosed as low risk for CAD by simply relying on their normal MPI result or on their traditional cardiac risk factors taking a further step in the risk stratification and optimal management of these patients.

The prospective study of Choudhary et al which is published in the current issue of the Journal addressed exactly the above-mentioned issue of further risk stratification of patients with normal MPI scans who might benefit from aggressive medical therapy using CACS and CTA. ${ }^{13}$ The patient population included 81 mostly symptomatic ( $81 \%$ ) patients (only three of them women) with mean age of $60.4 \pm 9.6$ years and without prior history of CAD or abnormal stress test who had normal results on stress MPI [either by exercise (48 patients) or dobutamine (33 patients) stress protocols]. All of the patients underwent CACS and 16-slice CTA within 2 to 4 weeks of the MPI. Cardiac risk estimates by ATP III criteria and post-MPI probability of CAD were also calculated for each patient. As a result of the study, 43 patients (53\% of all patients) were identified as candidates for aggressive medical therapy using a post-test risk stratification model which classified patients as having a high risk, when at least one the following three conditions was present: either diabetes or ATP III 10 year-risk estimate of $>20 \%$; CACS $>400$; or coronary stenosis of $>50 \%$ on CTA. While only one of these criteria was met in 29 patients, 7 patients shared all of 
the three criteria and another 14 had both high CACS and significant stenosis on CTA. Lowering the cutoff value of CACS to 100 resulted in almost complete detection (sensitivity $96 \%$ ) of patients with $>50 \%$ stenosis on CTA, but lowered the specificity from $91 \%$ to $61 \%$. CTA provided further incremental risk stratification over CACS in nine patients who had $>50 \%$ stenosis on CTA and CACS of $<400$. However, the most striking of all findings was that MPI failed to identify $28 \%$ of patients with significant obstructive stenosis on CTA in a mostly symptomatic cohort of patients with predominantly intermediate to high 10 -year risk for cardiac events.

Authors went further and designed a risk stratification algorithm based on their findings, which aimed to provide more optimal utilization of these prognostic modalities. The first step of their algorithm required identification of patients with diabetes and high ATP III risk scores $(>20 \%)$ which would directly qualify for aggressive medical therapy. Further risk stratification with CACS was proposed for patients with either intermediate ATP III risk score (10-20\%) or intermediate post-test probability. Those with CACS above 400 would be directed to aggressive medical treatment arm and those with CACS in the range of 100 to 399 would be referred for further evaluation by CTA. At the final step patients with $>50 \%$ coronary stenosis on CTA would be identified as high risk and treated aggressively with plaque stabilizing medical therapy.

This is an important study that highlights the limitation of MPI in defining patients who might benefit from further therapy and close outpatient monitoring. These findings are in concordance with the fact that changes in the coronary anatomy and histology always precede myocardial ischemia. Consequently, lack of ischemia does not necessarily preclude the presence of significant atherosclerotic burden. Moreover, myocardial ischemia can be considered as a late sign in the atherosclerotic process, correction of which does not really result in a permanent "cure". Hence, it is important to look for ways of early detection and treatment of patients with atherosclerosis long before the occurrence of myocardial ischemia. Thus, the findings of the present study should promote further quest for more optimal post-MPI risk stratification and management algorithms.

Even though, the overall structure of the proposed risk stratification algorithm seems to be quite reasonable, it should also be stressed on the point that the management of symptomatic patients with normal MPI results does not necessarily have to be limited solely to medical therapy. With the addition of CTA to the algorithm, clinicians should feel confident enough to pursue further invasive imaging by coronary catheterization, especially if anti-anginal therapy proves to be insufficient in patients with symptoms and $>50 \%$ stenosis on CTA. After all, symptomatic relief is one of the few undisputable benefits of coronary revascularization.

Despite it is conceivable that based on the available evidence presented at recently published trials the assumptions about the high risk criteria used in the proposed post-test risk stratification algorithm must be theoretically correct, they still need to be validated in larger scale randomized prognostic studies testing for this particular algorithm. Moreover, further research is also needed to accurately determine the "warranty" period of a normal MPI in different risk groups of patients, so that the patients who might need closer monitoring and re-assessment by either repeated MPI testing or other imaging modalities can be accurately determined.

It is also worthwhile mentioning the limitations of this study that might prevent the generalization of its findings. First, this was a single-center study in which the participants were referred for stress MPI by a multispecialty group of physicians. As the investigators also noted, this practice pattern might not exactly reflect the common type of medical management applied to the general population. Second, the number of patients in this cohort was relatively small requiring caution in interpretation of results. There was also a marked gender imbalance, so that women constituted less than $4 \%$ of the population, limiting the applicability of the conclusions only to men. The exclusion of patients with prior history of CAD from the study was appropriate because that group of patients is expected by all means to be managed with aggressive medical therapy regardless of the MPI result.

In conclusion, the findings of this study show that a normal MPI result is not the end of the road for risk stratification of patients with symptoms. Substantial portion of patients could still be categorized as high risk based on the presence of diabetes, high 10-year risk on ATP III, CAC score $>400$ or a $>50 \%$ coronary stenosis on CTA. Further research is needed to define the most appropriate risk stratification algorithm that would utilize the best of the qualities of each risk assessment tool and imaging modality in most optimal order.

\section{Open Access}

This article is distributed under the terms of the Creative Commons Attribution Noncommercial License which permits any noncommercial use, distribution, and reproduction in any medium, provided the original author(s) and source are credited. 


\section{References}

1. Shaw LJ, Iskandrian AE. Prognostic value of gated myocardial perfusion SPECT. J Nucl Cardiol 2004;11:171-85.

2. Hachamovitch R, Hayes S, Friedman JD, et al. Determinants of risk and its temporal variation in patients with normal stress myocardial perfusion scans-What is the warranty period of a normal scan? J Am Coll Cardiol 2003;41:1329-40.

3. Rumberger JA, Simons DB, Fitzpatrick LA, Sheedy PF, Schwartz RS. Coronary artery calcium area by electron-beam computed tomography and coronary atherosclerotic plaque area: A histopathologic correlative study. Circulation 1995;92:2157-62.

4. Sangiorgi G, Rumberger JA, Severson A, et al. Arterial calcification and not lumen stenosis is highly correlated with atherosclerotic plaque burden in humans: A histologic study of 723 coronary artery segments using nondecalcifying methodology. J Am Coll Cardiol 1998;31:126-33.

5. Ramakrishna G, Miller TD, Breen JF, et al. Relationship and prognostic value of coronary artery calcification by electron beam computed tomography to stress-induced ischemia by single photon emission computed tomography. Am Heart J 2007;153:807-14.

6. Uebleis C, Becker A, Griesshammer I, et al. Stable coronary artery disease: Prognostic value of myocardial perfusion SPECT in relation to coronary calcium scoring-long term follow up. Radiology 2009;252:682-90.

7. Budoff MJ, Rasouli L, Shavelle DM, et al. Cardiac CT angiography (CTA) and nuclear myocardial perfusion imaging (MPI)—a comparison in detecting significant coronary artery disease. Acad Radiol 2007;14:252-7.

8. Schuijf JD, Wijns W, Jukema JW, et al. Relationship between noninvasive coronary angiography with multi-slice computed tomography and myocardial perfusion imaging. J Am Coll Cardiol 2006;48:2508-14

9. Gaemperli O, Schepis T, Valenta I, et al. Functionally relevant coronary artery disease: Comparison of 64-section CT angiography with myocardial perfusion SPECT. Radiology 2008;248:414-23.

10. Danciu SC, Herrera CJ, Stecy PJ, et al. Usefulness of multislice computed tomographic coronary angiography to identify patients with abnormal myocardial perfusion stress in whom diagnostic catheterization may be safely avoided. Am J Cardiol 2007;100: 1605-8.

11. van Werkhoven JM, Schuijf JD, Gaemperli O, et al. Prognostic value of multislice computed tomography and gated single-photon emission computed tomography in patients with suspected coronary artery disease. J Am Coll Cardiol 2009;53:623-32.

12. Johnson KM, Dowe DA, Brink JA. Traditional clinical risk assessment tools do not accurately predict coronary atherosclerotic plaque burden: A CT angiography study. AJR 2009;192:235-43.

13. Choudhary G, Shin V, Punjani S, Ritter N, Sharma S, Wu W. The role of calcium score and CT angiography in the medical management of patients with normal myocardial perfusion imaging. $\mathrm{J}$ Nucl Cardiol 2010. doi:10.1007/s12350-009-9158-x. 